\title{
Caristi-Type Fixed Point Theorem over Száz Principle in Quasi-Metric Space with a Graph
}

\author{
Karim Chaira, ${ }^{1}$ Abderrahim Eladraoui $\mathbb{D}^{1,2}{ }^{1,2}$ Mustapha Kabil, ${ }^{2}$ and Samih Lazaiz $\mathbb{D}^{3}$ \\ ${ }^{1}$ Laboratory of Algebra, Analysis and Applications, Faculty of Sciences Ben M'sik, University of Hassan II Casablanca, Morocco \\ ${ }^{2}$ Laboratory of Mathematics and Applications, Faculty of Sciences and Technologies of Mohammedia, \\ University of Hassan II Casablanca, Morocco \\ ${ }^{3}$ Laboratory of Mathematical Analysis and Applications (LAMA), Faculty of Sciences Dhar El Mahraz, \\ University Sidi Mohamed Ben Abdellah, Fes, Morocco \\ Correspondence should be addressed to Abderrahim Eladraoui; a.adraoui@live.fr
}

Received 17 February 2019; Accepted 10 June 2019; Published 1 July 2019

Academic Editor: Basil K. Papadopoulos

Copyright (C) 2019 Karim Chaira et al. This is an open access article distributed under the Creative Commons Attribution License, which permits unrestricted use, distribution, and reproduction in any medium, provided the original work is properly cited.

The aim of this paper is to generalize Caristi's fixed point theorem in a K-complete quasi-metric space endowed with a reflexive digraph by using Száz maximum principle. An example is given to support our main result.

\section{Introduction}

Let $X$ be a nonempty set. A binary relation " $\leqslant$ " on $X$ is said to be a preorder on $X$ if it is reflexive and transitive. In this case $(X, \preccurlyeq)$ is called a preordered set. An element $x \in X$ is said to be maximal in $X$ if for all $y \in X$,

$$
x \preccurlyeq y \Longrightarrow x=y .
$$

The set $S_{\leqslant}^{+}(x)=\{z \in X ; x \leqslant z\}$ is called the final segment generated by $x$.

In 2007, Á. Száz (see [1]) generalized the Brézis-Browder principle in the setting of the preorder sets and gave a generalized version of Caristi's theorem.

Theorem 1 (Száz [1]). Let $(X, \preccurlyeq)$ be a preordered set and let $\Phi: X \times X \longrightarrow \overline{\mathbb{R}}$ be a function satisfying:

(S1) $x \longmapsto \sup _{y \in S_{\gtrless}^{+}(x)} \Phi(x, y)$ is decreasing;

(S2) $-\infty<\sup _{y \in S_{\leftarrow}^{+}(x)} \Phi(x, y)$ for all $x \in X$;

(S3) $\sup _{y \in S_{\aleph}^{+}(a)} \Phi(a, y)<\infty$ for some $a \in X$;
(S4) For every nondecreasing sequence $\left\{x_{n}\right\}_{n \in \mathbb{N}} \subset X$ with $x_{0}=a$, there exists some $x \in X$ such that $x_{n} \leqslant x$ for all $n \in \mathbb{N}$ and $\liminf _{n \rightarrow \infty} \Phi\left(x_{n}, x_{n+1}\right)=0$;

(S5) $0<\Phi(x, y)$ for all $x, y \in X$ with $x<y$.

Then, there exists a maximal element $\hat{x} \in X$.

In 1976, Caristi (see [2]) gave a generalization of Banach contraction principle where the assumption " $T: X \longrightarrow X$ is continuous" is dropped and replaced by a weak assumption. Since then, various proofs, extensions, and generalizations are given by many authors (see [3-7]). It is worth mentioning that Caristi's fixed point theorem is equivalent to the Ekeland's variational principle [8].

In this work, we use the Száz principle to give a more generalized version of Caristi's fixed point theorem in the setting of the quasimetric space with a graph. For that, we introduce a new class of functions called $K$-functions and $M$ functions which generalize the notion of dominated function in Caristi's theorem. Also, we give an improved result in the framework of set-valued mappings and we derive some known results as corollaries. 


\section{Preliminaries}

Definition 2. Let $X$ be a nonempty set; a function $\delta: X \times$ $X \longrightarrow[0, \infty)$ is quasidistance if we have

(1) $\delta(x, y)=0$ if and only if $x=y$

(2) $\delta(x, z) \leq \delta(x, y)+\delta(y, z)$ for each $x, y, z \in X$.

The pair $(X, \delta)$ is called a quasimetric space.

Since $\delta(x, y)=\delta(y, x)$ is not necessarily satisfied in such spaces, there are many characterizations of completeness in this setting (e.g., [9]). Following the framework of [8], we have the following.

Definition 3. A sequence $\left\{x_{n}\right\}_{n}$ in $(X, \delta)$ is

(1) left K-Cauchy if for every $\varepsilon>0$ there exists $n_{0} \in \mathbb{N}$ such that $\forall n, m \in \mathbb{N}$, with $n_{0} \leq n \leq m, \delta\left(x_{n}, x_{m}\right)<\varepsilon$;

(2) left K-converges to $x$, if $\lim _{n \rightarrow \infty} \delta\left(x, x_{n}\right)=0$;

(3) $(X, \delta)$ will be called left $\mathrm{K}$-complete quasimetric if any left K-Cauchy sequence is left $\mathrm{K}$-convergent.

Definition 4. Let $(X, \delta)$ be a quasimetric space. A mapping $\varphi: X \longrightarrow \mathbb{R}_{+}$is said to be

(1) lower semicontinuous if given any sequence $\left\{x_{n}\right\}_{n}$ in $X$, whenever $\lim _{n} x_{n}=\bar{x}$ and $\lim _{n} \varphi\left(x_{n}\right)=r$, then $\varphi(\bar{x}) \leq r ;$

(2) upper semicontinuous if $-\varphi$ is lower semicontinuous.

In the sequel, we recall some basic notions on graphs borrowed from [10].

Let $V$ be an arbitrary set. A directed graph, or digraph, is a pair $G=(V, E)$ where $E$ is a subset of the Cartesian product $V \times V$. The elements of $V$ are called vertices or nodes of $G$ and the elements of $E$ are the edges, also called oriented edges or arcs of $G$. An edge of the form $(v, v)$ is a loop on $v$. Another way to express that $E$ is a subset of $V \times V$ is to say that $E$ is a binary relation over $V$. Given a digraph $G$, the set of vertices (respectively, of edges) of $G$ is denoted by $V(G)$ (respectively, $E(G))$. The digraph $G=(V, E)$ is said to be

(i) transitive if whenever $(x, y) \in E$ and $(y, z) \in E$, then $(x, z) \in E$

(ii) reflexive if $\Delta:=\{(v, v) \mid v \in V\}$ is a subset of $E$.

A vertex $x$ is said to be isolated if for all vertex $y \neq x$, we have neither $(x, y) \in E$ nor $(y, x) \in E$. Given two vertices $x, y \in V$. A path in $G$, from (or joining) $x$ to $y$ is a sequence of vertices $p=\left\{a_{i}\right\}_{0 \leq i \leq n}, n \in \mathbb{N}^{*}$ such that $a_{0}=x, a_{n}=y$ and $\left(a_{i}, a_{i+1}\right) \in E$, for all $i \in\{0,1, \ldots, n-1\}$. The integer $n$ is the length of the path $p$. If $x=y$ and $n>1$, the path $p$ is called a directed cycle. An acyclic digraph is a digraph which has no directed cycle.

We denote by $y \in[x]_{G}$ the fact that there is a directed path in $G$ joining $x$ to $y$.

A quasimetric space $(X, \delta)$ endowed with a digraph $G$ such that $V(G)=X$ is denoted by $(X, \delta, G)$.

As in [4], we use the following.
Definition 5. Let $(X, \delta, G)$ be a quasimetric space endowed with a digraph. We say that $X$ satisfies the property (OSC) if for any sequence $\left\{x_{n}\right\} \subseteq X$ that is convergent to $x \in X$ and for all $n \in \mathbb{N}, x_{n+1} \in\left[x_{n}\right]_{G}$, then $x \in\left[x_{n}\right]_{G}$ for all $n \in \mathbb{N}$.

Let $(X, G)$ be a reflexive digraph, and a function $\Phi$ : $X \times X \longrightarrow \overline{\mathbb{R}}$. Having in mind $\Phi(x, y)=\varphi(x)-\varphi(y)$ for each $x, y \in X$, most dominated Caristi's functions satisfied the following conditions:

(C1) N-superadditivity: $\Phi(x, x)=0$ and $\Phi(x, y)+$ $\Phi(y, z) \leq \Phi(x, z)$ for each $x, y, z \in X$ with $y \in[x]_{G}$ and $z \in[y]_{G}$.

(C2) $y \longmapsto \Phi(x, y)$ is upper semicontinuous for each $x \in$ $X$.

(C3) There exists $a \in X$ such that $\sup _{y \in[a]_{G}} \Phi(a, y)<\infty$.

(C4) There exists a function $\psi: X \longrightarrow \mathbb{R}$ such that, for all $x, y \in X$,

$$
y \in[x]_{G} \Longrightarrow \Phi(x, y) \leq \psi(x) .
$$

Next, we introduce a new class of functions.

Definition 6. Let $(X, G)$ be a reflexive digraph; a real function $\Phi: X \times X \longrightarrow \overline{\mathbb{R}}$ is said to be

(i) K-function if (C1), (C2), and (C3) hold.

(ii) $M$-function if (C1), (C2), and (C4) hold.

Remark 7. Obviously, each $M$-function is a $K$-function. Indeed, let $a \in X$. Then for each $y \in[a]_{G}$, we have $\Phi(a, y) \leq$ $\psi(a)$ which implies

$$
\sup _{y \in[a]_{G}} \Phi(a, y) \leq \psi(a)<\infty .
$$

\section{Main Results}

Let $(X, \delta, G)$ be a quasimetric space endowed with a digraph. Define a binary relation on $X$ by

$$
x \preccurlyeq_{\Phi} y \Longleftrightarrow\left\{\begin{array}{l}
\delta(x, y) \leq \Phi(x, y) \\
y \in[x]_{G} .
\end{array}\right.
$$

We will use particularly the following fact.

Lemma 8. Let $(X, \delta, G)$ be a quasimetric space with a digraph and $\Phi: X \times X \longrightarrow \overline{\mathbb{R}}$ a function satisfying condition (C1); then $\left(X, \delta, \preccurlyeq_{\Phi}\right)$ is a preordered quasimetric space.

The following result is a generalization and an extension of Caristi's theorem in the setting of the quasimetric spaces with a graph.

Theorem 9. Let $(X, \delta, G)$ be a left $K$-complete quasimetric space endowed with a reflexive digraph satisfying the (OSC) property and $T: X \longrightarrow 2^{X}$ a set-valued map. If there exists a K-function $\Phi: X \times X \longrightarrow \overline{\mathbb{R}}$ such that for each $x \in X$, there exists $y \in T x \cap[x]_{G}$ with $\delta(x, y) \leq \Phi(x, y)$, then $T$ has a fixed point in $X$. 


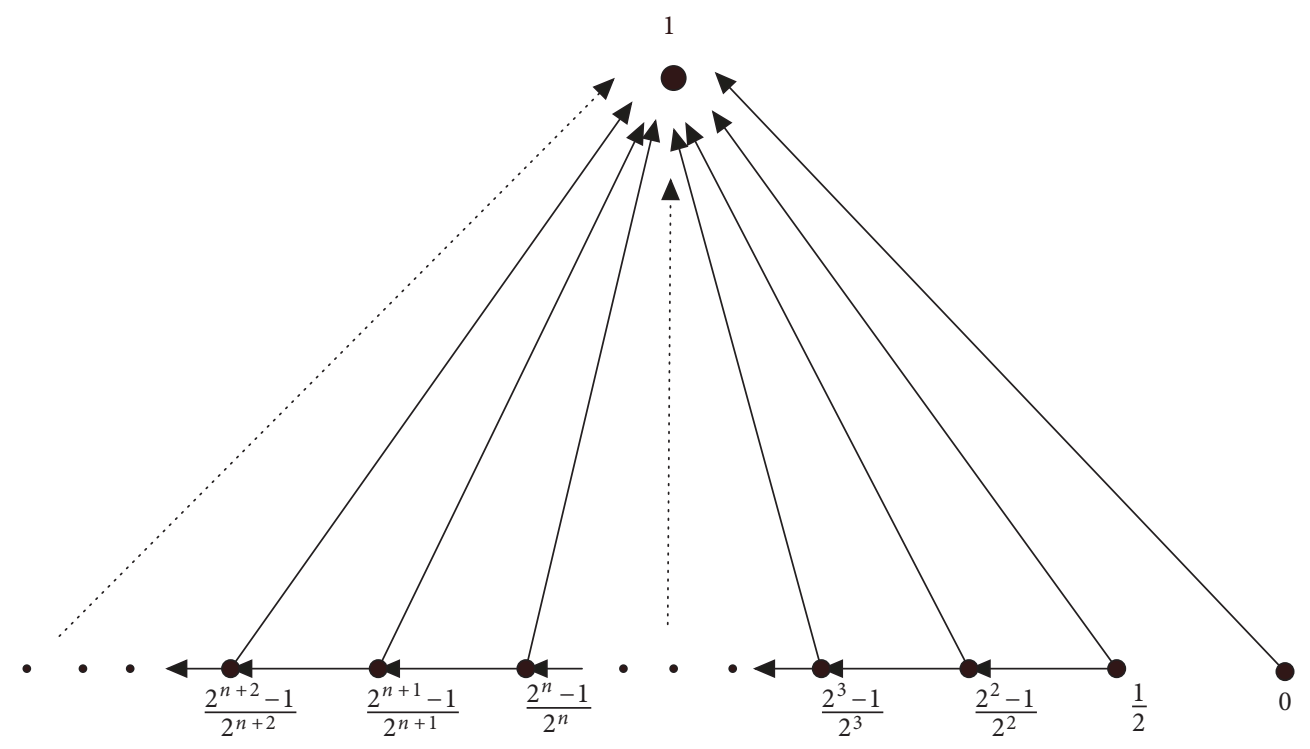

FIGURE 1: Graph G (the loops and the isolated vertices are not represented).

Proof. Firstly, we show that for each increasing sequence $\left\{x_{n}\right\}_{n}$ with respect to $\preccurlyeq_{\Phi}$ where $x_{0}=a$, there exists $\bar{x}$ such that for each $n \in \mathbb{N}$ we get $x_{n} \preccurlyeq_{\Phi} \bar{x}$.

According to the definition of $\preccurlyeq_{\Phi}$, we have $x_{n} \preccurlyeq_{\Phi} x_{n+1} \Longrightarrow$ $\delta\left(x_{n}, x_{n+1}\right) \leq \Phi\left(x_{n}, x_{n+1}\right)$; hence,

$$
\begin{aligned}
\sum_{i=0}^{n} \delta\left(x_{i}, x_{i+1}\right) & \leq \sum_{i=0}^{n} \Phi\left(x_{i}, x_{i+1}\right) \leq \Phi\left(a, x_{n+1}\right) \\
& \leq \sup _{y \in S_{\aleph_{\Phi}}^{+}(a)} \Phi(a, y)
\end{aligned}
$$

Then $\sum_{i=0}^{n} \Phi\left(x_{i}, x_{i+1}\right)$ is real convergent sequence, so $\lim _{n \rightarrow \infty} \Phi\left(x_{n}, x_{n+1}\right)=0$. And $\left\{x_{n}\right\}_{n}$ is a left K-Cauchy sequence in $X$ since $\sum_{i=0}^{\infty} \delta\left(x_{i}, x_{i+1}\right)$ is convergent. Then, $\left\{x_{n}\right\}_{n}$ left K-converges to some $\bar{x}$. The property (OSC) guarantees that $\bar{x} \in\left[x_{n}\right]_{G}$ for all $n \in \mathbb{N}$.

Note that for each $n, m \in \mathbb{N}$ with $n \leq m$, we get

$$
x_{n} \preccurlyeq_{\Phi} x_{m} \Longrightarrow \delta\left(x_{n}, x_{m}\right) \leq \Phi\left(x_{n}, x_{m}\right)
$$

which by left K-convergence of $\left\{x_{n}\right\}_{n}$ and upper semicontinuity of $y \longmapsto \Phi(x, y)$, we have $\lim \sup _{m \rightarrow \infty} \Phi\left(x_{n}, x_{m}\right) \leq$ $\Phi\left(x_{n}, \bar{x}\right)$. Then, $\delta\left(x_{n}, \bar{x}\right) \leq \Phi\left(x_{n}, \bar{x}\right)$ and $\bar{x} \in\left[x_{n}\right]_{G}$ for all $n \in \mathbb{N}$, which leads to

$$
x_{n} \preccurlyeq_{\Phi} \bar{x}, \quad \text { for every } n \in \mathbb{N} .
$$

Thus, (S4) holds.

Define a function $\varphi: X \longrightarrow[0, \infty]$ for each $x \in X$ by

$$
\varphi(x)=\sup _{y \in[x]_{G}} \Phi(x, y)
$$

$\varphi$ is nonnegative function, since for each $x \in X$, there is $y \in$ Tx such that $\delta(x, y) \leq \Phi(x, y)$; then $0 \leq \varphi(x)$ that is (S2) holds.

If $x \prec_{\Phi} y$; that is, $\left(x \preccurlyeq_{\Phi} y\right.$ and $\left.x \neq y\right)$, then $0<\delta(x, y) \leq$ $\Phi(x, y)$, which implies (S5).
Let $x \preccurlyeq_{\Phi} y$; then for each $z \in[y]_{G}$, we get

$$
\Phi(x, y)+\Phi(y, z) \leq \Phi(x, z)
$$

and since $\Phi(x, y) \geq 0$, we have

$$
\Phi(y, z) \leq \Phi(x, z)
$$

Then

$$
\sup _{z \in[y]_{G}} \Phi(y, z) \leq \sup _{z \in[x]_{G}} \Phi(x, z)
$$

i.e., $\varphi(y) \leq \varphi(x)$, which implies that $\varphi$ is nonincreasing function; that is, (S1) holds.

All assumptions of Száz principle hold; then $X$ has a maximal element $\hat{x}$. By hypothesis, there exists $\hat{y} \in T \hat{x}$ such that $\widehat{x} \preccurlyeq_{\Phi} \hat{y}$; then we get $\widehat{x}=\hat{y}$, which implies that $\widehat{x} \in T \widehat{x}$.

We support our result by the following example.

Example 10. Consider the digraph $G=(X, E)$ represented in Figure 1, where

$$
\begin{aligned}
X= & \{0,1\} \cup\left\{1-\frac{1}{2^{n}}: n \in \mathbb{N}\right\}, \\
E= & \Delta \cup\{(0,1)\} \\
& \cup\left\{\left(1-\frac{1}{2^{n}}, 1\right),\left(1-\frac{1}{2^{n}}, 1-\frac{1}{2^{n+1}}\right): n \in \mathbb{N}^{*}\right\}
\end{aligned}
$$

Define on $X$ the quasimetric $\delta$ as follows:

$$
\delta(x, y)= \begin{cases}y-x & \text { if } y \in[x]_{G} \\ 1 & \text { ifnot. }\end{cases}
$$

Consider the $K$-function $\Phi: X \times X \longrightarrow \overline{\mathbb{R}}$ defined by

$$
\Phi(x, y)=\ln ^{2}\left(\frac{y+1}{x+1}\right)+y-x
$$


and the set-valued mapping $T: X \longrightarrow 2^{X}$ defined by

$$
\begin{aligned}
T(0) & =\{1\} ; \\
T\left(1-\frac{1}{2^{n}}\right) & =\left\{1-\frac{1}{2^{n^{k}}}: k \in \mathbb{N}\right\} ; \\
T(1) & =X .
\end{aligned}
$$

One can see that

(i) $x \in[1]_{G}$ for all $x \in X$.

(ii) For all $n, m \in \mathbb{N}^{*}$ with $n>m$, we have $1-1 / 2^{n} \in$ $\left[1-1 / 2^{m}\right]_{G}$.

(iii) $(X, d)$ is a complete left $K$-quasimetric space.

(iv) $\Phi$ is obviously $K$-function.

(v) For all $x \in X$, there exists $y \in T x \cap[x]_{G}$ such that

$$
d(x, y) \leq \ln ^{2}\left(\frac{y+1}{x+1}\right)+y-x
$$
$T(1 / 2)$.

All assumptions of Theorem 9 are satisfied and $1 / 2 \epsilon$

Corollary 11. Under assumptions of Theorem 9, with $T$ : $X \longrightarrow X$ is only a single-valued map and for all $x \in X$,

$$
T x \in[x]_{G} \Longrightarrow \delta(x, T x) \leq \Phi(x, T x),
$$

then $T$ has a fixed point in $X$.

Using Remark 7, we have immediately the following.

Corollary 12. Under assumptions of Theorem 9, if $\Phi: X \times$ $X \longrightarrow \overline{\mathbb{R}}$ is an $M$-function, then $T$ has a fixed point in $X$.

The following theorem improves the results of $[2,11,12]$ and generalizes the main theorem of Chaira et al. [4]

Theorem 13. Let $(X, \delta, G)$ be a left $K$-complete quasimetric space with a reflexive digraph satisfying the (OSC) property and $\varphi: X \longrightarrow[0, \infty)$ a lower semicontinuous function. If the mapping $T: X \longrightarrow X$ satisfies, for all $x \in X$,

$$
T x \in[x]_{G} \Longrightarrow \delta(x, T x) \leq \varphi(x)-\varphi(T x)
$$

then $T$ has a fixed point in $X$.

Proof. We consider the function $\phi: X \times X \longrightarrow \overline{\mathbb{R}}$ defined by

$$
\phi(x, y)=\varphi(x)-\varphi(y), \quad \forall x, y \in X
$$

It is clear that $\phi$ is a K-function. Applying Theorem 9, the proof is complete.

\section{Data Availability}

There were no data used to support this study.

\section{Conflicts of Interest}

The authors declare that there are no conflicts of interest regarding the publication of this paper.

\section{References}

[1] Á. Száz, "An improved altman type generalization of the BrézisBrowder ordering principle," Mathematical Communications, vol. 12, no. 2, pp. 155-161, 2007.

[2] J. Caristi, "Fixed point theorems for mappings satisfying inwardness conditions," Transactions of the American Mathematical Society, vol. 215, pp. 241-251, 1976.

[3] M. Altman, "A generalization of the Brézis-Browder principle on ordered sets," Nonlinear Analysis: Theory, Methods \& Applications, vol. 6, no. 2, pp. 157-165, 1982.

[4] K. Chaira, A. Eladraoui, M. Kabil, and S. Lazaiz, "Extension of kirk-saliga fixed point theorem in a metric space with a reflexive digraph," International Journal of Mathematics and Mathematical Sciences, vol. 2018, Article ID 1471256, 6 pages, 2018.

[5] M. Jleli, B. Samet, C. Vetro, and F. Vetro, "From Caristi's theorem to Ekeland's variational principle in $0_{\sigma}$-complete metric like spaces," Abstract and Applied Analysis, vol. 2014, Article ID 319619, 7 pages, 2014.

[6] M. Turinici, "A generalization of Altman's ordering principle," Proceedings of the American Mathematical Society, vol. 90, no. 2, pp. 128-132, 1984.

[7] C. Vetro and F. Vetro, "Caristi type selections of multivalued mappings," Journal of Function Spaces, vol. 2015, Article ID 941856, 6 pages, 2015.

[8] S. Cobzaş, "Completeness in quasi-metric spaces and ekeland variational principle," Topology and Its Applications, vol. 158, no. 8, pp. 1073-1084, 2011.

[9] I. L. Reilly, P. V. Subrahmanyam, and M. K. Vamanamurthy, "Cauchy sequences in quasi-pseudo-metric spaces," Monatshefte für Mathematik, vol. 93, no. 2, pp. 127-140, 1982.

[10] M. Rigo, Advanced Graph Theory and Combinatorics, John Wiley \& Sons, 2016.

[11] E. Karapinar and S. Romaguera, "On the weak form of Ekeland's Variational Principle in quasi-metric spaces," Topology and Its Applications, vol. 184, pp. 54-60, 2015.

[12] S. Romaguera and P. Tirado, "A characterization of Smyth complete quasi-metric spaces via Caristi's fixed point theorem," Fixed Point Theory and Applications, vol. 2015, no. 1, pp. 1-13, 2015. 


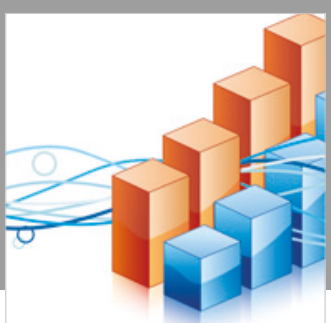

Advances in

Operations Research

\section{-n-m}
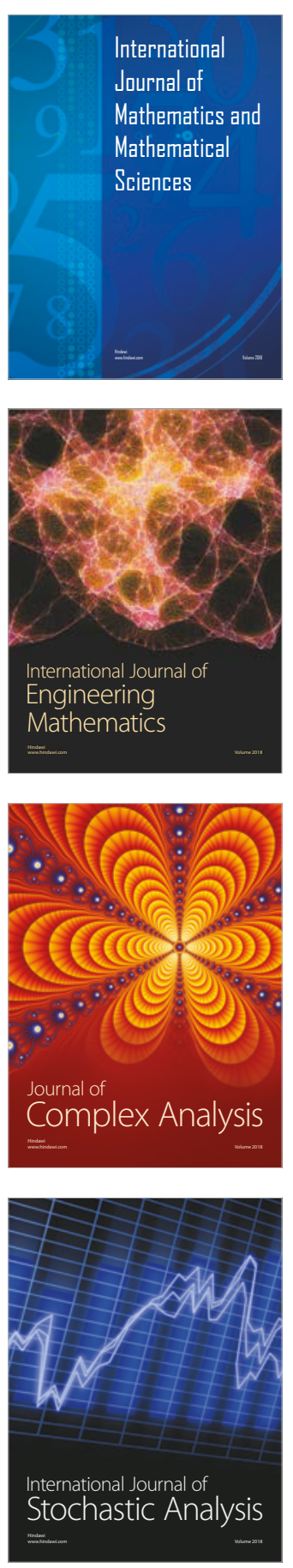
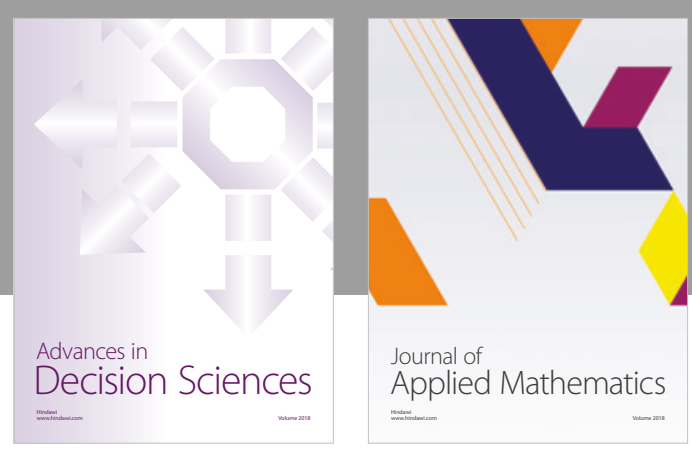

Journal of

Applied Mathematics
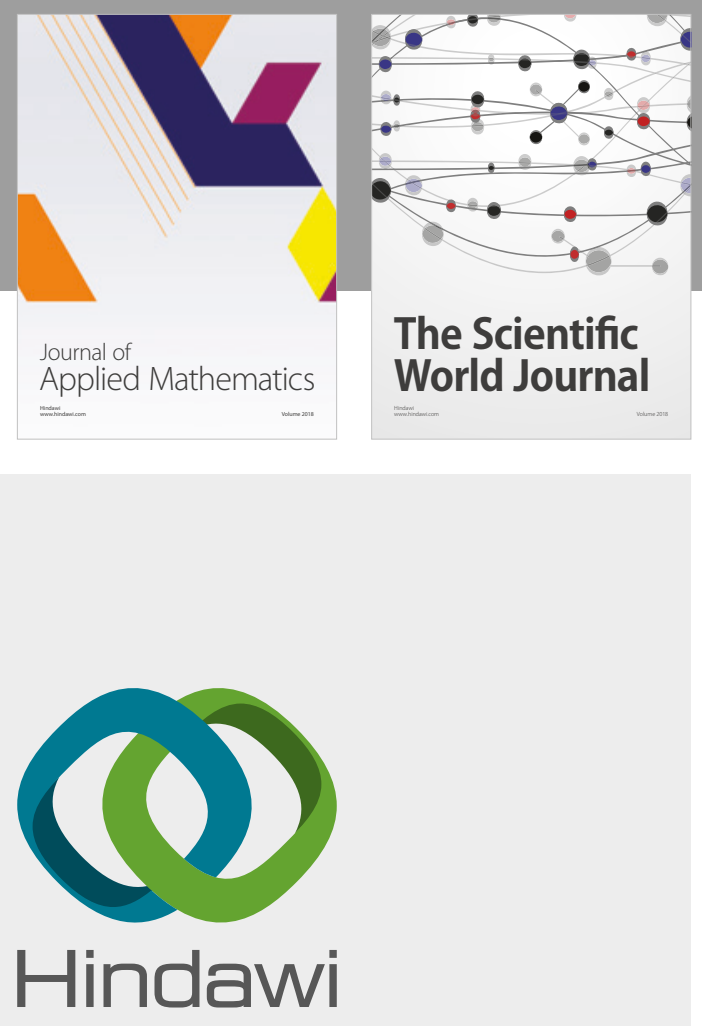

Submit your manuscripts at

www.hindawi.com

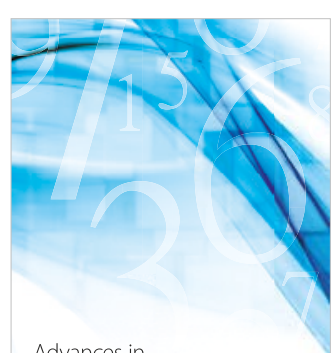

Advances in
Numerical Analysis
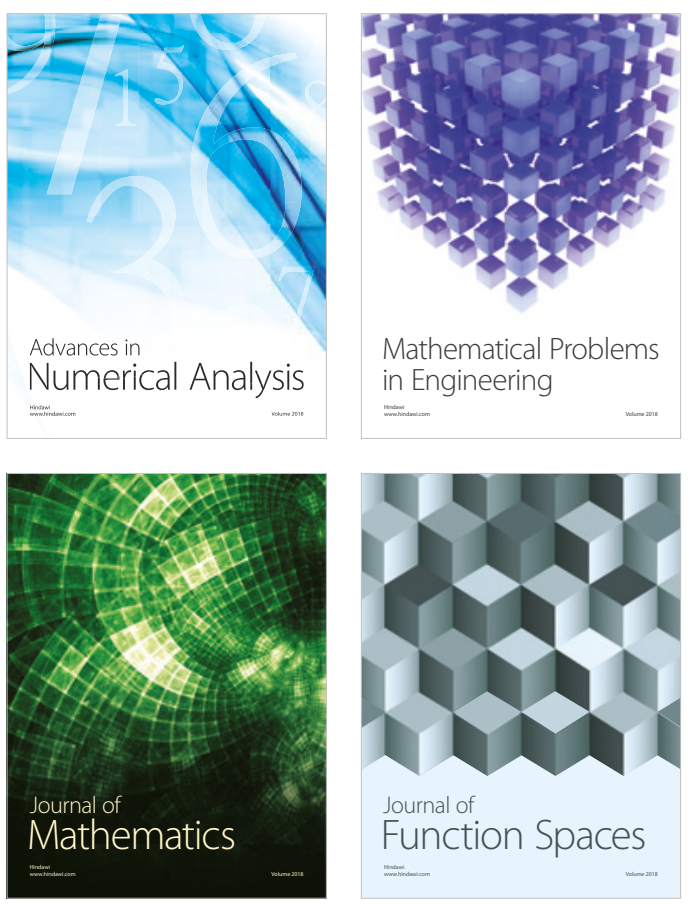

Mathematical Problems in Engineering

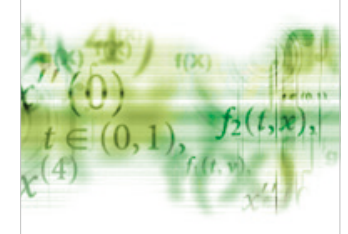

International Journal of

Differential Equations

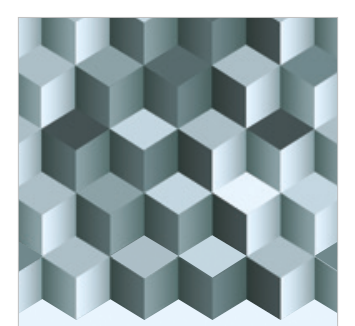

Journal of

Function Spaces
The Scientific

World Journal

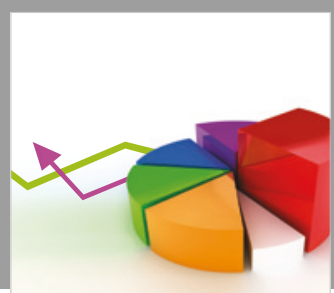

Journal of

Probability and Statistics
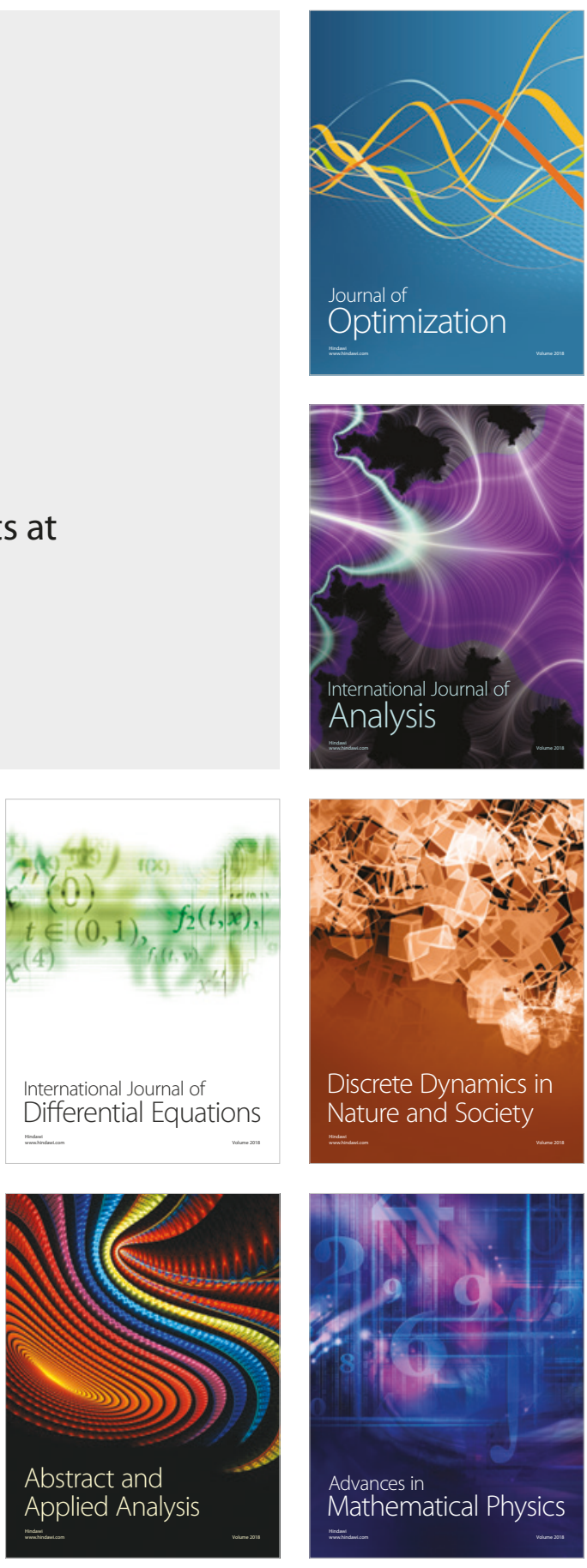